\title{
Pseudospin-lattice coupling in the spin-orbit Mott insulator $\mathrm{Sr}_{2} \mathrm{IrO}_{4}$
}

\author{
J. Porras, ${ }^{1}$ J. Bertinshaw, ${ }^{1}$ H. Liu, ${ }^{1}$ G. Khaliullin, ${ }^{1}$ N. H. Sung, ${ }^{1}$ J.-W. Kim, ${ }^{2}$ S. Francoual,${ }^{3}$ P. Steffens, ${ }^{4}$ G. Deng, ${ }^{5}$ \\ M. Moretti Sala,${ }^{6,7}$ A. Efimenko, ${ }^{6}$ A. Said, ${ }^{2}$ D. Casa, ${ }^{2}$ X. Huang, ${ }^{2}$ T. Gog, ${ }^{2}$ J. Kim, ${ }^{2}$ B. Keimer, ${ }^{1}$ and B. J. Kim ${ }^{1,8,9,}$ \\ ${ }^{1}$ Max-Planck-Institut für Festkörperforschung, Heisenbergstraße 1, 70569 Stuttgart, Germany \\ ${ }^{2}$ Advanced Photon Source, Argonne National Laboratory, Argonne, Illinois 60439, USA \\ ${ }^{3}$ Deutsches Elektronen-Synchrotron DESY, 22603 Hamburg, Germany \\ ${ }^{4}$ Institut Laue-Langevin 6, rue Jules Horowitz, Boîte Postale 156, 38042 Grenoble Cedex 9, France \\ ${ }^{5}$ Australian Nuclear Science and Technology Organization, Lucas Height, New South Wales 2233, Australia \\ ${ }^{6}$ European Synchrotron Radiation Facility, Boîte Postale 220, 38043 Grenoble Cedex, France \\ ${ }^{7}$ Dipartimento di Fisica, Politecnico di Milano, Piazza Leonardo da Vinci 32, 20133 Milano, Italy \\ ${ }^{8}$ Department of Physics, Pohang University of Science and Technology, Pohang 790-784, South Korea \\ ${ }^{9}$ Center for Artificial Low Dimensional Electronic Systems, Institute for Basic Science, 77 Cheongam-Ro, Pohang 790-784, South Korea
}

(Received 4 September 2018; revised manuscript received 10 November 2018; published 15 February 2019)

\begin{abstract}
Spin-orbit entangled magnetic dipoles, often referred to as pseudospins, provide a new avenue to explore novel magnetism inconceivable in the weak spin-orbit coupling limit, but the nature of their low-energy interactions remains to be understood. We present a comprehensive study of the static magnetism and low-energy pseudospin dynamics in the archetypal spin-orbit Mott insulator $\mathrm{Sr}_{2} \mathrm{IrO}_{4}$. We find that in order to understand even basic magnetization measurements, a formerly overlooked in-plane anisotropy is fundamental. In addition to magnetometry, we use neutron diffraction, inelastic neutron scattering, and resonant elastic and inelastic x-ray scattering to identify and quantify the interactions that determine the global symmetry of the system and govern the linear responses of pseudospins to external magnetic fields and their low-energy dynamics. We find that a pseudospin-only Hamiltonian is insufficient for an accurate description of the magnetism in $\mathrm{Sr}_{2} \mathrm{IrO}_{4}$ and that pseudospin-lattice coupling is essential. This finding should be generally applicable to other pseudospin systems with sizable orbital moments sensitive to anisotropic crystalline environments.
\end{abstract}

DOI: 10.1103/PhysRevB.99.085125

\section{INTRODUCTION}

The $4 d$ and $5 d$ transition-metal compounds are characterized by spin-orbit entangled and spatially extended valence electrons, which in magnetic insulators translate to strong and long-range interactions among pseudospins. Pseudospins, having sizable orbital contributions to the magnetic moment, are highly sensitive to the crystalline symmetry [1] and thus interact through multiple interactions whose hierarchy depends on the lattice geometry and the pseudospin quantum number. For example, pseudospins- $1 / 2$ in a honeycomb lattice have dipolarlike, bond-directional interactions, which dominate over isotropic (Heisenberg) interactions and constitute the key building block for the Kitaev spin liquid [2-6]. The opposite is true for a square lattice in which the leading-order interaction is isotropic, rendering a rare realization outside of the cuprate family of a (pseudo)spin- $1 / 2$ antiferromagnet on a square lattice [7-11]. Pseudospins-1 on the same lattice may be subject to a single-ion anisotropy that is much stronger than all nearest-neighbor (NN) interactions and leads to distinct physics characterized by "soft" magnetic moments supporting a Higgs amplitude mode [12,13].

On the experimental side, recent technological advances in resonant inelastic $x$-ray scattering (RIXS) [14] have

*bjkim6@postech.ac.kr allowed key insights into the nature of magnetism expressed by pseudospins through measurement of the momentumresolved dynamic structure factor. Extensive efforts in the last several years have revealed the nature of leading-order interactions in a number of strongly spin-orbit coupled materials: e.g., bond-directional interactions in $\mathrm{Na}_{2} \mathrm{IrO}_{3}$ [6], Heisenberg interactions in $\mathrm{Sr}_{2} \mathrm{IrO}_{4}[9,15,16]$, and Ising interactions in $\mathrm{Sr}_{3} \mathrm{Ir}_{2} \mathrm{O}_{7}[17,18]$. However, the limited energy resolution of RIXS has so far not allowed for substantial information beyond the leading-order interactions. Despite their smaller energy scales, next-order interactions play a crucial role in determining the magnetic phase of the system and its stability against perturbations. For instance, the Kitaev spin liquid phase has a finite window of stability when perturbed by Heisenberg interactions [3]. For magnetically ordered systems, the low-energy physics determines the global symmetry of the magnetic structure and thereby the topology of the electronic system as a whole. A prominent example is the pyrochlore iridates with the so-called all-in-all-out magnetic structure, which is a prerequisite for the Weyl semimetal phase predicted in $\mathrm{Nd}_{2} \mathrm{Ir}_{2} \mathrm{O}_{7}$ [19].

In this study, we use a comprehensive set of experimental techniques to overcome the limitations in determining the nature of the interactions governing the ground state of the prototypical quasi-two-dimensional square-lattice iridate $\mathrm{Sr}_{2} \mathrm{IrO}_{4}$. It is now well established that a $(\pi, \pi)$ staggered arrangement of pseudospins, also known as $J_{\text {eff }}=1 / 2$ moments [7], is 
(a)

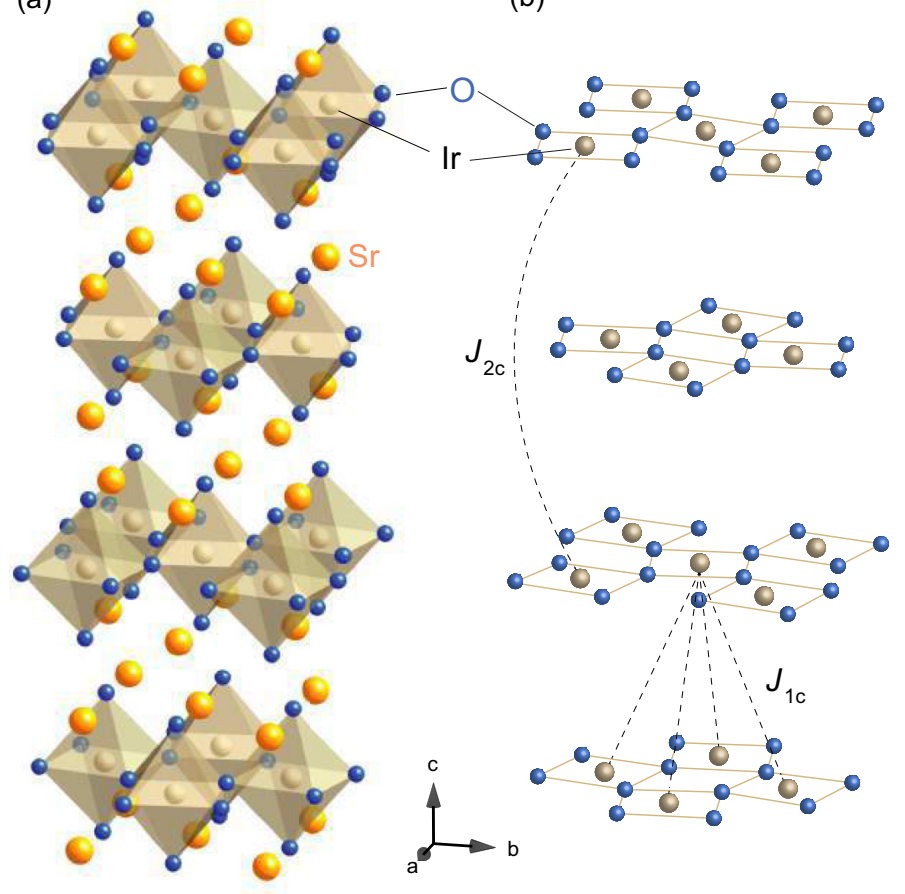

(c)

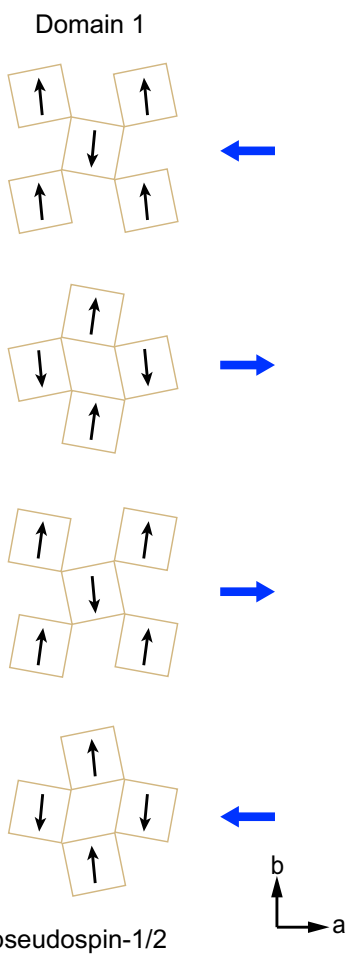

Domain 2
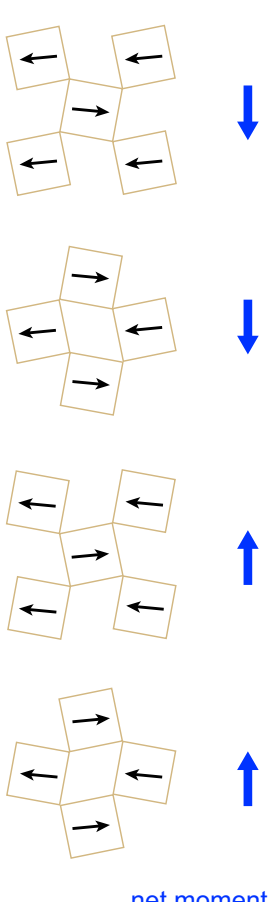

FIG. 1. (a) The crystal structure of $\mathrm{Sr}_{2} \mathrm{IrO}_{4}$ is tetragonal (space group $I 4_{1} / a$ ), with $a=b=5.49 \AA$ and $c=25.80 \AA$ at room temperature. The tetragonal $a$ and $b$ axes are rotated by $45^{\circ}$ from the $\mathrm{Ir}-\mathrm{O}$-Ir bond directions. Ir atoms lie in the center of oxygen octahedra. IrO $\mathrm{O}_{2}$ layers are separated by SrO layers. (b) Interlayer pseudospin couplings between the nearest layers and the next-nearest layers via $J_{1 \mathrm{c}}$ and $J_{2 \mathrm{c}}$, respectively. (c) Top view on the $\mathrm{IrO}_{2}$ planes, with arrows indicating canted pseudospins (black) and net ferromagnetic moments (blue), following the possible magnetic domain configurations in the $4_{1}$ crystal symmetry.

stabilized by the strong antiferromagnetic (AFM) NN Heisenberg interaction $(J \sim 60 \mathrm{meV}$ [9]). This state, which remains intact even when charge carriers are introduced by chemical $[15,16]$ or photodoping [20] to disrupt the static long-range order, underlies a striking parallel between the phenomenology of electron-doped $\mathrm{Sr}_{2} \mathrm{IrO}_{4}$ and hole-doped cuprates, namely, high-temperature pseudogaps and low-temperature $d$-wave gaps in the single-particle removal spectra [21-23]. The complex static long-range order (Fig. 1) that sets in at $T_{N} \simeq$ $230 \mathrm{~K}$ [24] reveals additional interactions at play, including anisotropic interactions that confine the pseudospins to the $a b$ plane, Dzyaloshinskii-Moriya (DM) interactions that cant the pseudospins and add up to a nonzero net moment in each $\mathrm{IrO}_{2}$ layer [2], and interlayer couplings that stabilize the "upup-down-down" (uudd) stacking pattern of the net moments along the $c$ axis $[8,25,26]$ (see Fig. 2).

These interactions manifest as a deviation from the Heisenberg universality class evidenced by the temperature dependence of the order parameter in diffuse $\mathrm{x}$-ray scattering [10,27], a resonance line in electrons spin resonance [28], and a spin-wave gap in Raman scattering [29,30] and RIXS [16]. However, interpretations of these experiments have led to mutually inconsistent results, and a coherent understanding of the low-energy pseudospin dynamics is still lacking. For instance, the energy scale for the out-of-plane spin-wave gap, a direct measure of the magnetic anisotropy, inferred from these measurements varies widely between $\lesssim 1 \mathrm{meV}$ [28] and $30 \mathrm{meV}$ [16]. The lack of knowledge about the hierarchy among these interactions is an impediment to our understanding of the mechanism that stabilizes the observed static magnetic structure, notably, the fact that the magnetic easy axis points away from the NN bonds [Fig. 1(c)].

In this paper, we present a comprehensive study of the low-energy pseudospin interactions that generate the static magnetic structure and govern linear responses to magnetic fields of the archetypal spin-orbit Mott insulator $\mathrm{Sr}_{2} \mathrm{IrO}_{4}$. Our work establishes a minimal Hamiltonian that captures the full three-dimensional static magnetic structure and uncovers the essential role of pseudospin-lattice coupling thus far overlooked in most theories of magnetism in strongly spin-orbit coupled materials. Our work has important implications for all experiments involving quantities that depend on the global symmetry and/or topology of the system, such as the selection rules for the second-harmonic generation [31-33]. Further, it raises the question of the role of lattice degrees of freedom in emergent phases of the square-lattice iridates [21-23,31,32], which can serve as a model system for electron-lattice interactions in many other correlated electron materials such as the colossal magnetoresistive manganites [34] and hightemperature superconducting cuprates [35].

\section{GROUND STATE AND LOW-FIELD MAGNETISM}

\section{A. Magnetic domains}

We start by discussing all possible magnetic domain configurations and their evolution in magnetic fields in order to 


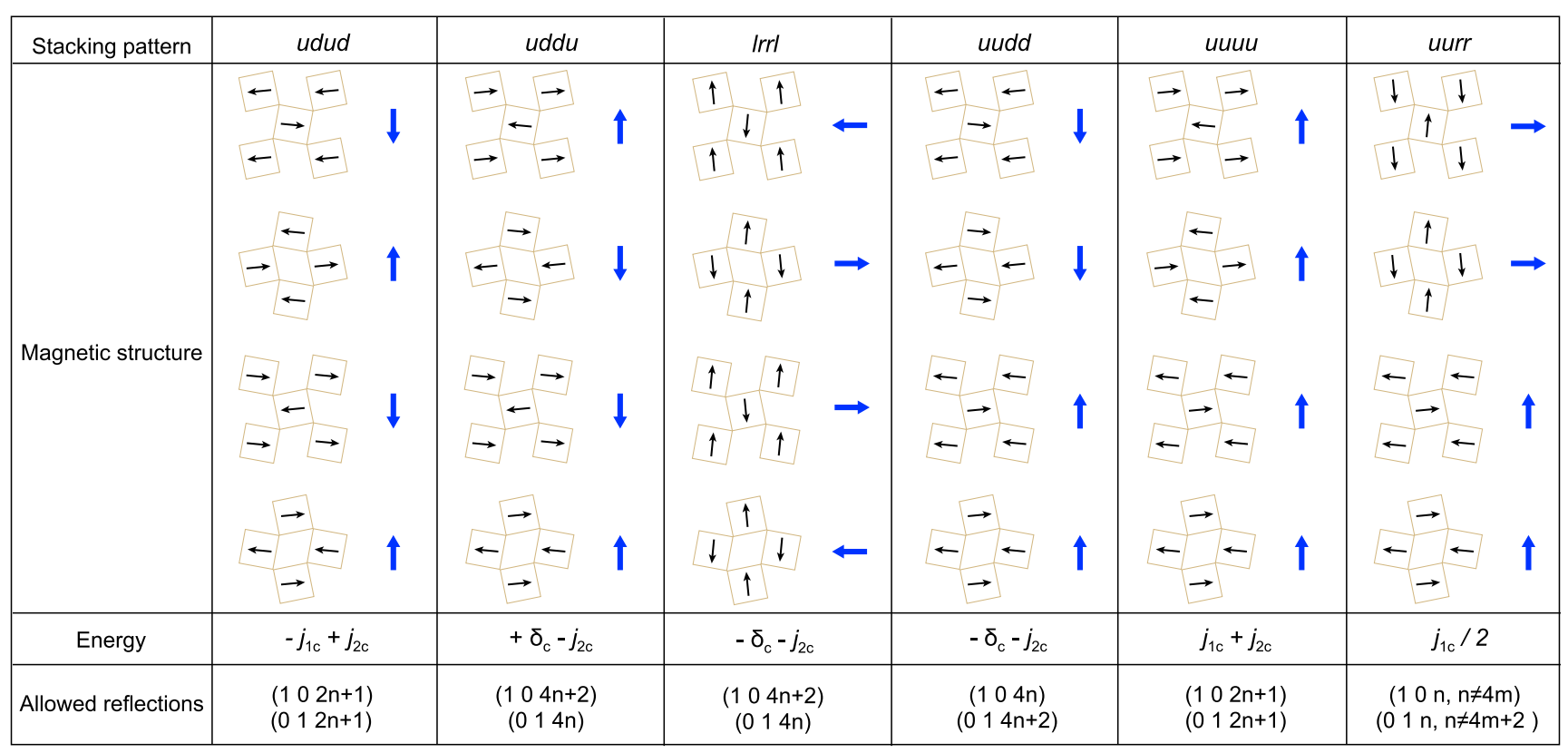

FIG. 2. Top view of possible stacking of pseudospins (black arrows) and the corresponding net ferromagnetic moment (blue arrows) in each layer, where the labeling up $(u)$, down $(d)$, left $(l)$, and right $(r)$ refers to their orientation in the $a b$ plane. The energy difference between each of these configurations and the ground state of Eq. (1) and its allowed reflections are indicated at the bottom. The energy is written in terms of the effective couplings between the net moments $j_{1 c}=4 S^{2} J_{1 c} \sin ^{2} \phi, j_{2 c}=-S^{2} J_{2 c}\left(\cos ^{2} \phi-\sin ^{2} \phi\right)$, and $\delta_{c}=4 S^{2} \Delta_{c} \cos ^{2} \phi$, where $\phi$ is the canting angle. Here $u d d u$ or $u u d d$ is stabilized when $j_{2 \mathrm{c}}>0$ and $\left|j_{1 \mathrm{c}}\right|<2 j_{2 \mathrm{c}}$. With $\delta_{c}>0 u u d d$ or $l r r l$ becomes more favorable than $u d d u$ (or llrr, not shown).

disentangle the response from a single domain. The magnetic ordering breaks the $4_{1}$ screw-axis symmetry of the crystal structure of $\mathrm{Sr}_{2} \mathrm{IrO}_{4}$ (fourfold rotation about the $c$ axis followed by the one quarter translation along the same lattice vector), which means that successive $4_{1}$ operations generate four possible magnetic domains. Only two of these can be distinguished macroscopically as the other two are different only by up $\leftrightarrow$ down sublattice switching of the Néel order. Thus, there are two distinguishable domains: one with the pseudospins mostly along the $b$ axis with $l r r l$ stacking of the canted ferromagnetic component and the other along the $a$ axis with uudd stacking [see Figs. 1(c) and 2]. As discussed later, the correlation between the pseudospin direction and the stacking pattern necessitates inclusion of an anisotropic interlayer coupling [see Fig. 5(b)], which is symmetry allowed and should be generally nonzero.

In our resonant magnetic x-ray scattering (RMXS) experiments, shown in Figs. 3(a) and 3(c), the two domains are visible as two distinct reflections, (llll $\left.\begin{array}{lll}0 & 1 & 24\end{array}\right)$ and $\left(\begin{array}{lll}0 & 1 & 26\end{array}\right)$, owing to their two different stacking patterns. When corrected by geometrical and polarization factors, the intensities of the two reflections directly measure the population of the two domains, which we follow as a function of applied magnetic field. The results agree reasonably well with a simulation assuming 50:50 domain population, as shown in Figs. 3(b) and $3(\mathrm{~d})$.

With increasing magnetic field applied along the [010] direction [Figs. 3(a) and 3(b)] the domain with pseudospins along [100] shrinks as the domain with pseudospins along [010] grows. This can be simply understood since there is a Zeeman energy gain from the net ferromagnetic moments induced along the field, giving rise to the $\left(\begin{array}{lll}0 & 1 & 25\end{array}\right)$ reflec- tion, but the domain repopulation involves complex domain wall motions reflected as deviations from linear behavior and

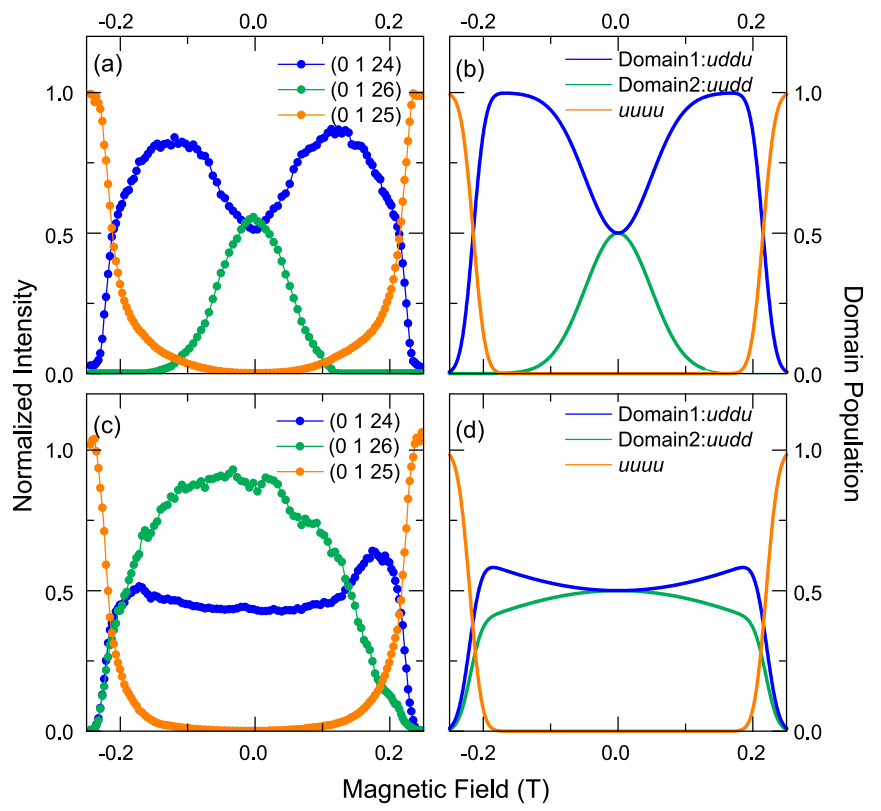

FIG. 3. Normalized RMXS intensity of magnetic reflections $\left(\begin{array}{lll}0 & 1 & 24\end{array}\right),\left(\begin{array}{lll}0 & 1 & 25\end{array}\right)$, and (llll $\left.\begin{array}{lll}0 & 1 & 26\end{array}\right)$ as a function of magnetic field applied along (a) [010] and (c) [110], compared to simulated domain populations shown in (b) and (d), respectively. In (d), the applied field was slightly tilted away from [110] to mimic the misalignment of the field in experimental conditions. The data were taken at $T=60 \mathrm{~K}$, and the intensity was corrected for structure and polarization factors. At around 0.2 T the stacking pattern changes to ииии (see Fig. 2). 
(a)
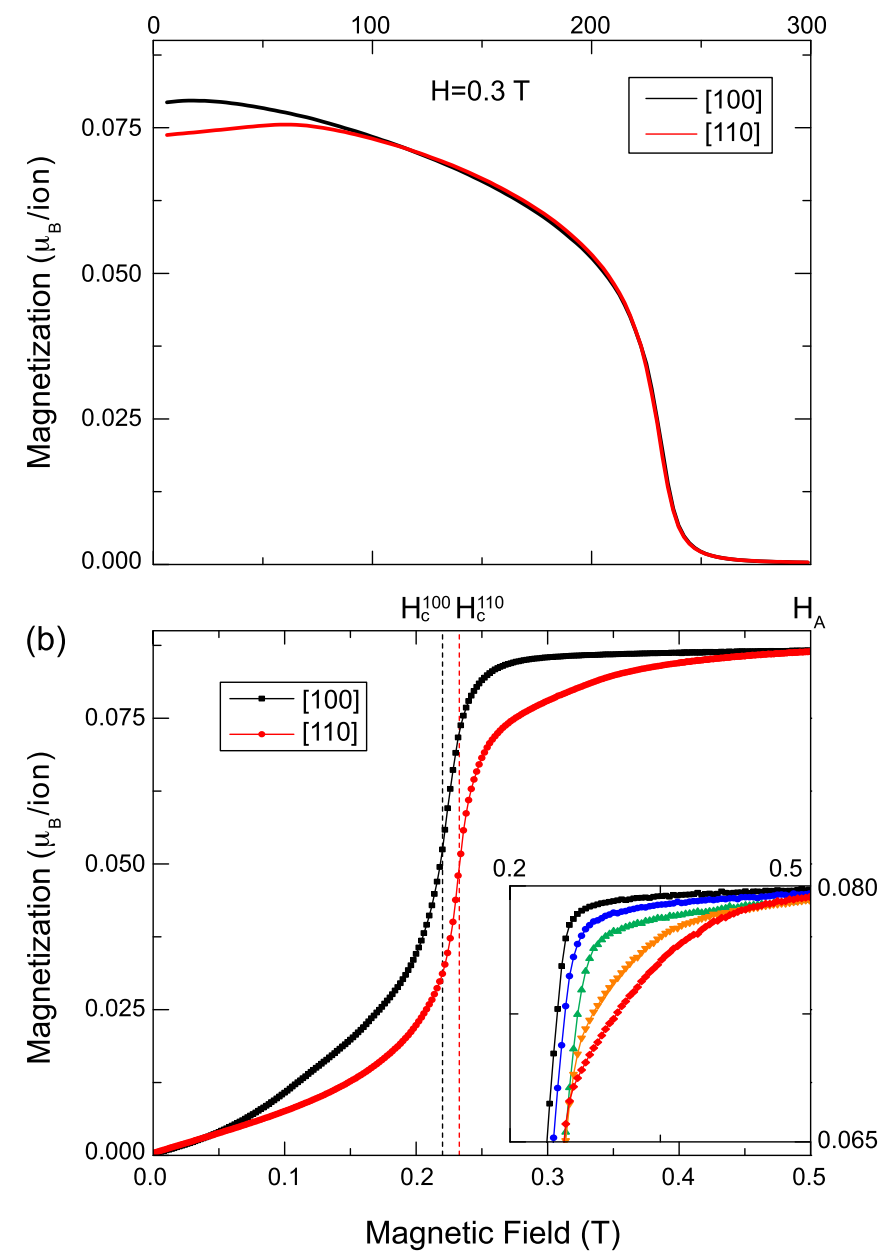

FIG. 4. (a) Magnetization as a function of temperature along [100] (black) and [110] (red), taken at $H=0.3$ T. (b) Magnetization as a function of magnetic field for [100] (black) and [110] (red), taken at $T=5 \mathrm{~K}$. Inset: detailed measurements for different field angles from [100] to [110] every $11.25^{\circ}$, focusing on the region where the biggest effect due to anisotropy is seen.

hysteresis in the magnetization measurements shown later [Fig. 4(b)]. At $\simeq 0.1 \mathrm{~T}$, the magnetic domains are fully aligned, as can be seen from the vanishing intensity of $\left(\begin{array}{lll}0 & 1 & 26\end{array}\right)$ and the saturation of $\left(\begin{array}{lll}0 & 1 & 24\end{array}\right)$. For fields $H>0.2 \mathrm{~T}$, the intensity of the $\left(\begin{array}{lll}0 & 1 & 25\end{array}\right)$ reflection probing ииии stacking (Fig. 2) greatly increases, while $\left(\begin{array}{lll}0 & 1 & 24\end{array}\right)$ decreases, indicating a metamagnetic transition where the ferromagnetic moments align with the field.

When the field is applied along [110] [Figs. 3(c) and 3(d)], both domains remain populated at $\simeq 0.1 \mathrm{~T}$ as the field has no preference for either of the two domains. Any slight misalignment of the field from the [110] direction leads to an imbalance in the domain population, as can be seen from the small difference in the field dependence of the $\left(\begin{array}{lll}0 & 1 & 24\end{array}\right)$ and (llll 1126$)$ reflections. The persistence of both domains above $0.1 \mathrm{~T}$ implies an anisotropy within the $a b$ plane; without it the ferromagnetic moments would simply rotate perpendicular to the field.

\section{B. In-plane magnetic anisotropy}

We investigate the in-plane magnetic anisotropy by performing longitudinal magnetization measurements. Figure 4(a) shows the magnetization as a function of temperature with a magnetic field applied along [100] and [110]. The magnetization shows an upturn at $T_{\mathrm{N}} \simeq 230 \mathrm{~K}$, where the system becomes antiferromagnetic. The black curve for measurements along [100] shows an order-parameter-like increase that persists to the lowest temperature, which is characteristic of the weak ferromagnetism, as the applied field of $H=$ $0.3 \mathrm{~T}$ is enough to fully align the net moments along [100] [Fig. 3(b)]. For the measurements along [110] shown in red, a decrease in the magnetization is observed at low temperature, which points to a temperature-dependent anisotropy. We note that it requires a very high quality sample to observe the in-plane anisotropy as it was not visible in previous magnetization measurements [see the Supplemental Material [36] for a description of our samples].

In order to understand the origin of such anisotropy, the magnetization as a function of magnetic field was studied at $T=5 \mathrm{~K}$. In Fig. 4(b), anisotropic behavior below $0.5 \mathrm{~T}$ is clearly seen. In particular, (i) the two curves for magnetic field along [100] and [110] have different slopes below $0.2 \mathrm{~T}$; (ii) a metamagnetic transition occurs at $H_{c}^{100}=0.22 \mathrm{~T}$ and $H_{c}^{110}=$ $0.23 \mathrm{~T}$, respectively; and (iii) saturation in the magnetization is attained slightly above $H_{c}^{100}$ along [100] but only at a higher field $H_{A} \simeq 0.5 \mathrm{~T}$ along [110]. The inset shows measurements at different angles between these two limits, showing the gradual change from one behavior to the other.

For a quantitative analysis of the magnetization measurements, it is necessary to consider the possible mechanisms for in-plane anisotropy.

\section{Mechanisms for in-plane anisotropy}

As previously discussed, the Hamiltonian for magnetic interactions in $\mathrm{Sr}_{2} \mathrm{IrO}_{4}$ is dominated by Heisenberg interactions:

$$
H_{\mathrm{iso}}=\sum_{\langle i j\rangle} J_{i j} \vec{S}_{i} \cdot \vec{S}_{j}+J_{1 \mathrm{c}} \vec{S}_{i} \cdot \vec{S}_{j}+J_{2 \mathrm{c}} \vec{S}_{i} \cdot \vec{S}_{j}
$$

where $\vec{S}_{i}$ labels the pseudospin at site $i$ and $J_{i j}$ denote first $(J)$, second $\left(J_{2}\right)$, and third $\left(J_{3}\right)$ in-plane nearest-neighbor interactions [9]. Similarly, $J_{1 \mathrm{c}}$ and $J_{2 \mathrm{c}}$ are the first- and second-nearest-interlayer interactions, as shown in Fig. 1(b). The nearest-layer term $J_{1 \mathrm{c}}$ is partially frustrated due to the staggering of pseudospins in adjacent layers, as pointed out in an earlier study [37]. The next-nearest-layer term $J_{2 \mathrm{c}}$ is responsible for the $u u d d$ and $l r r l$ stacking patterns (Fig. 2).

Additionally, tetragonal distortion and rotation of octahedra lead to symmetric and antisymmetric exchange anisotropy terms of the form:

$$
H_{\mathrm{ani}}^{(1)}=\sum_{\langle i j\rangle} J_{z} S_{i}^{z} S_{j}^{z}+\vec{D} \cdot\left(\vec{S}_{i} \times \vec{S}_{j}\right)
$$

where $\vec{D}$ is the DM vector along the $c$ axis and gives rise to the canting angle $\phi$. The Hamiltonian (2) has been discussed in detail [38] in the context of $\mathrm{K}_{2} \mathrm{~V}_{3} \mathrm{O}_{8}$ [39]. $\mathrm{In}_{\mathrm{Sr}_{2}} \mathrm{IrO}_{4}$, these 

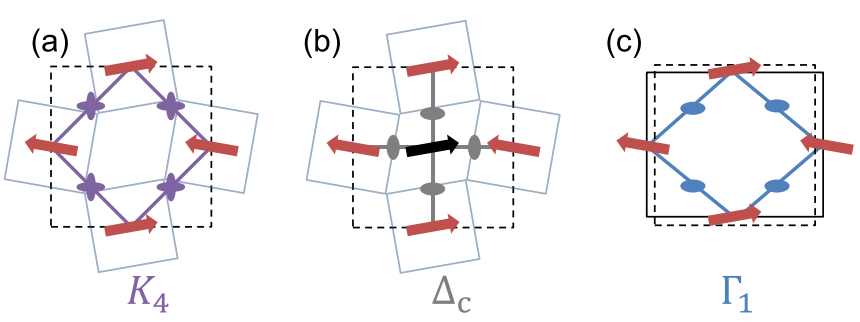

FIG. 5. An illustration of possible mechanisms for anisotropy. (a) Biaxial in-plane anisotropy $K_{4}$, shown as purple ellipses, (b) anisotropy in the out-of-plane nearest-neighbor coupling (gray ellipses) connecting pseudospins in two neighboring layers (red and black), and (c) anisotropy $\Gamma_{1}$ (blue ellipses) due to coupling of the pseudospins to the orthorhombically deformed lattice.

anisotropy terms confine the pseudospins to the $a b$ plane and give rise to an out-of-plane magnon gap [2].

The anisotropy within the $a b$ plane is naturally expected as a square lattice has only a discrete, fourfold rotation symmetry. Indeed, it has been observed in recent magnetoresistance [40] and torque magnetometry $[41,42]$ measurements. In the latter, a phenomenological biaxial anisotropy energy with magnetic easy axes along the crystallographic $a$ and $b$ axes of the form

$$
-K_{4} \cos 4 \theta
$$

was considered and is depicted in Fig. 5(a). $\theta$ is the angle between the canted ferromagnetic moments and $a$. Theoretically, biaxial anisotropy is attained when considering quantum order-by-disorder effects [43,44].

Another contribution to anisotropy comes from the anisotropic interlayer interaction [44]. This can be written as a $4{ }_{1}$ symmetry-allowed Hamiltonian:

$$
H_{\mathrm{ani}}^{(2)}=\sum_{\langle i j\rangle} \pm \Delta_{c}\left(S_{i}^{a} S_{j}^{a}-S_{i}^{b} S_{j}^{b}\right),
$$

where $\langle i j\rangle$ run over first-nearest neighbors in adjacent layers and $H_{\mathrm{ani}}^{(2)}$ changes sign depending on the direction of the bond [see Fig. 5(b)]. This term lifts the degeneracy between uudd (lrrl) and $u d d u(l l r r)$ and accounts for the observed magnetic structure: for the domain with the pseudospins mostly along the $a$ axis, $u u d d$ stacking is favored, whereas for the domain with the pseudospins mostly along the $b$ axis, $l r r l$ stacking is preferred [Fig. 1(c)].

In the model put forward by recent theoretical work [45], the coupling of the pseudospins to the lattice is responsible for the alignment of the moments along the crystallographic $a$ or $b$ direction and gives rise to in-plane anisotropy, as depicted in Fig. 5(c). It takes the form

$$
\begin{aligned}
H_{\text {sp-lat }}= & \sum_{\langle i j\rangle} \Gamma_{1} \cos 2 \theta\left(S_{i}^{x} S_{j}^{y}+S_{i}^{y} S_{j}^{x}\right) \\
& -\Gamma_{2} \sin 2 \theta\left(S_{i}^{x} S_{j}^{x}-S_{i}^{y} S_{j}^{y}\right),
\end{aligned}
$$

where $x$ and $y$ denote the directions along the Ir-O bonds and $\Gamma_{1}$ and $\Gamma_{2}$ are the energy scales of the pseudospin-lattice coupling to distortions along [100] and [110], respectively, scaled by the elasticity parameters and the square of the ordered moment. We note that while $H_{\mathrm{sp}-\text { lat }}$ preserves the four- fold symmetry per se, it leads to an orthorhombic distortion below $T_{N}$ and thus generates a uniaxial twofold anisotropy [45]. A special feature of this model is that the magnetic anisotropy potential is a function of the moment direction itself [via angle $\theta$ in Eq. (5)]. This is markedly different from the conventional, constant anisotropy terms $K_{4}$ and $\Delta_{c}$ discussed above.

We have calculated the ground-state configuration in an applied magnetic field and magnetization curves for the anisotropic Hamiltonians discussed above. Figure 6 shows the results for a biaxial anisotropy [Eq. (3); Fig. 6(a)], an anisotropy in the interlayer coupling [Eq. (4); Fig. 6(b)], a phenomenological uniaxial anisotropy of the form $-K_{2} \cos 2 \theta$ [Fig. 6(c)], and an anisotropy due to spin-lattice coupling following Eq. (5) [Fig. 6(d)] for a set of parameters (indicated in the caption) that best matches the data at $T=5 \mathrm{~K}$. These different types of anisotropies are schematically shown in the inset of each panel.

In Fig. 6(a), we consider the case for biaxial anisotropy. When the field is applied along the $a$ axis, in the favorable domain with moments along the $b$ axis as discussed above, the magnetization increases almost linearly at first, followed by a sudden jump to saturation at some critical field, as the net moments snap to the $a$ axis by the biaxial anisotropy. For field applied along the [110] direction, it takes much higher field to saturate the magnetization as the field has to overcome the biaxial anisotropy. However, it is important to note that a jump in the magnetization occurs at a lower field along the hard axis because there is an intermediate uurr phase (Fig. 2) that gains more than one half of the saturation Zeeman energy but does not cost any biaxial anisotropy energy. This is a key feature of the biaxial anisotropy model that differentiates it from the pseudospin-lattice coupling model. The former fails to correctly describe the data in Fig. 4(b) as $H_{c}^{110}<H_{c}^{100}$ for any set of values of the parameters.

In Fig. 6(b), the bare effect of the anisotropy in the out-ofplane coupling on the magnetization measurements is shown. When the field is applied along [100], the net moments cant toward the field, resulting in a linear increase of the total magnetization until the net moments are fully aligned along the field. For field along [110], the magnetization deviates from linear behavior; for small fields this direction is harder but becomes easier at some intermediate field compared to [100]. Although this type of anisotropy does not describe our magnetization data at low temperatures, it becomes relevant at higher temperatures, as shown in the next section.

For an illustration, we consider in Fig. 6(c) a hypothetical situation where the net moments are stabilized along the $b$ axis by a single-axis anisotropy, as one would expect if the tetragonal symmetry is reduced to orthorhombic, for instance, via uniaxial strain. When the field is applied along the hard $a$ axis, the magnetization is again linear. However, when the field is applied along the easy $b$ axis, the net moments remain in their zero-field orientation up to some field before they flop perpendicular to the field and then cant toward the field. This happens when the Zeeman energy gain overcomes the single-axis anisotropy. Note that the field along the $b$ axis required to saturate the magnetization is lower than that for the field along the $a$ axis because the single-axis anisotropy helps alignment along the $b$ axis. 


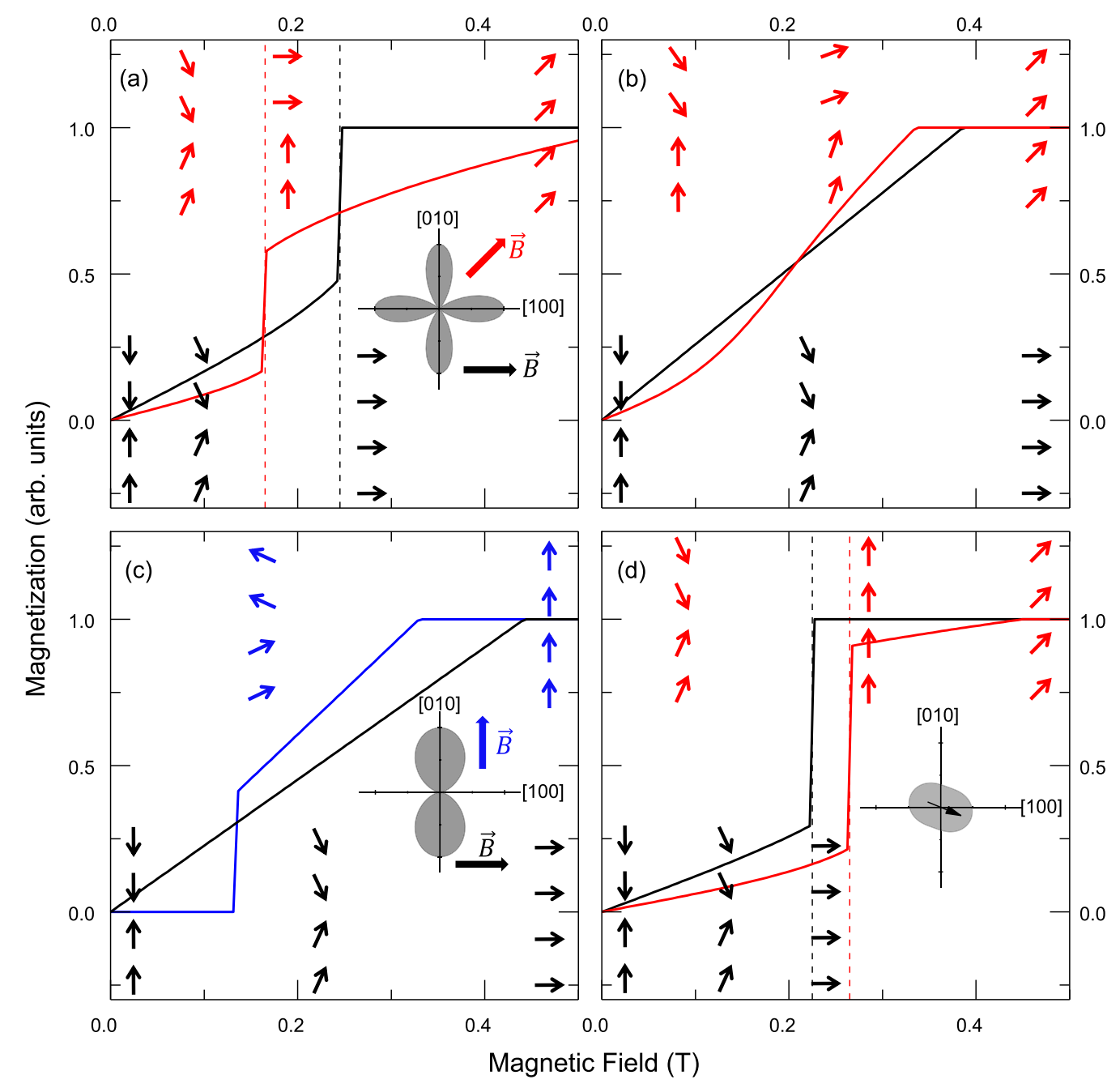

FIG. 6. Model calculation of the magnetization as a function of magnetic field applied along [100] (black) and [110] (red) for (a) biaxial anisotropy $K_{4}$, (b) anisotropy in the interlayer coupling $\Delta_{c}$, (d) anisotropy due to spin-lattice coupling $\Gamma_{1}$, and (c) uniaxial anisotropy along the hard [100] (black) and easy [010] (blue) axes. The parameters used are (a-d) $J_{1 \mathrm{c}}=16.4 \mu \mathrm{eV}$ and $J_{2 \mathrm{c}}=-6.2 \mu \mathrm{eV}$, (a), (b), and (d) $\Delta_{c}=$ $0.02 J_{1 \mathrm{c}}$, (a) $K_{4}=2.7 \mu \mathrm{eV}$, (c) $K_{2}=2.7 \mu \mathrm{eV}$, and (d) $\Gamma_{1}=2.7 \mu \mathrm{eV}$ and $\Gamma_{2}=0$. The moment orientations for different field configurations are shown as colored arrows. The insets in (a), (c), and (d) show schematically the in-plane anisotropy energy. Note that in (d) the anisotropy rotates as the moment does.

Finally, we consider the pseudospin-lattice coupling model in Fig. 6(d). It is clear that it captures the salient features of the data, in particular the fact that $H_{c}^{100}<H_{c}^{110}$. As in the case of the biaxial anisotropy model when the field is applied along the $a$ axis, the magnetization jumps to saturation because the $a$ axis and $b$ axis are equally energetically favorable. However, once the moment is aligned along a certain direction and the lattice is distorted along that direction, the pseudospin-lattice coupling effectively acts like a single-axis anisotropy, which means that the uurr type of stacking is never favored. For an applied field along [110], the moments flop to a ииии stacking pattern along either the $a$ or $b$ axis, before rotating toward the field direction.

\section{Further evidence for pseudospin-lattice coupling}

To further test the pseudospin-lattice coupling model, we performed RMXS measurements with polarization analysis and a 14-T split-coil cryomagnet at the P09 beamline at
DESY (see [36] for details). The experimental setup is shown in Fig. 7(a): $\pi$-polarized x rays scatter off the $\mathrm{Sr}_{2} \mathrm{IrO}_{4}$ single crystal with magnetic field applied along the [110] direction. Analysis of the polarization allows us to separate magnetic scattering from net moments along $[110]\left(\pi-\sigma^{\prime}\right)$ and $[1 \overline{1} 0]\left(\pi-\pi^{\prime}\right)$ (the main AFM components of the pseudospins are opposite). The uurr structure at intermediate fields between $H_{c}^{110}$ and $H_{A}$ in the biaxial anisotropy model can be described as a superposition of $u u d d$ stacking of moments along [11̄0] and ииии stacking of moments along [110], giving rise to signals from the $(4 \overline{5} 26)$ reflection in $\left(\pi-\pi^{\prime}\right)$ and $(4 \overline{5} 27)$ in $\left(\pi-\sigma^{\prime}\right)$, respectively, as depicted in Fig. 7(b). In contrast, in the pseudospin-lattice coupling model the moments align along the easy axis with ииии stacking, giving rise to signals from (4 $\overline{5}$ 27) reflection in both polarization channels but no signal in (4 $\overline{5}$ 26), as shown Fig. 7(c). Our measured data shown in Fig. 7(d) are in perfect agreement with the pseudospin-lattice coupling model. Together with the magnetization study, these results unambiguously establish that the in-plane anisotropy 
(a)

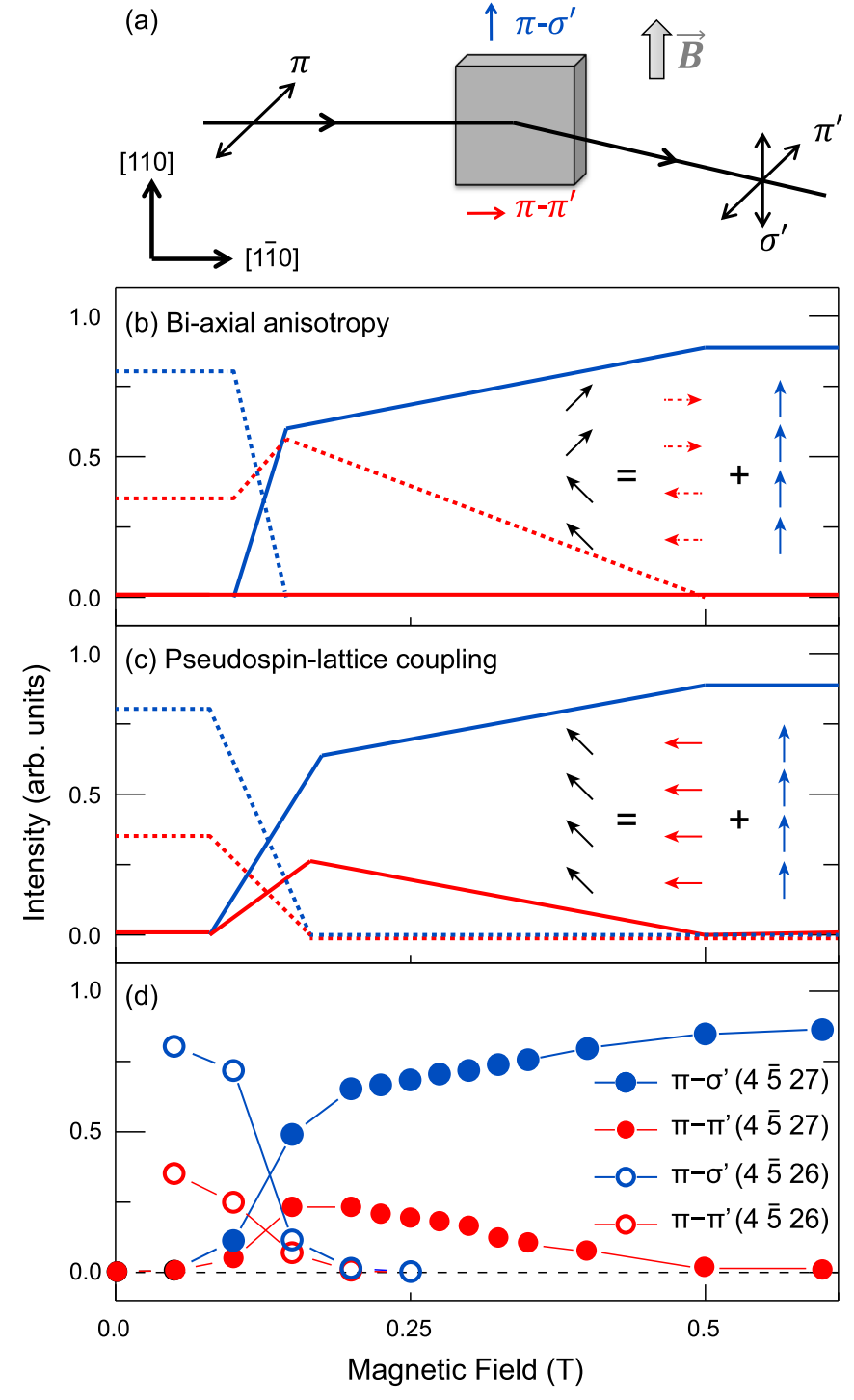

FIG. 7. (a) Schematic of the scattering geometry of the RMXS experiment: $\left(\pi-\sigma^{\prime}\right)\left[\left(\pi-\pi^{\prime}\right)\right]$ is sensitive to domains with net moment along [110] ([1 $\overline{1} 0])$. Calculated intensity of (4 $\overline{5}$ 26) and (4 $\overline{5} 27)$ magnetic reflections for the two polarizations as a function of field for the moment configuration attained with (b) biaxial anisotropy and (c) anisotropy due to spin-lattice coupling compared to the (d) measured integrated intensity taken at $T=5 \mathrm{~K}$. The insets in (b) and (c) show the characteristic moment configuration in the intermediatefield region, which can be separated into two components.

cannot be explained by only pseudospin interactions and requires a mechanism that breaks the fourfold symmetry of the underlying lattice, as provided by pseudospin-lattice coupling.

Next, we study the temperature dependence of the anisotropy. Figure 8 shows the magnetization as a function of field measured at various temperatures. As temperature increases, both the saturation field $H_{A}$ and the critical field $H_{c}^{110}$ decrease, with $H_{c}^{110}$ becoming smaller than $H_{c}^{100}$ around $100 \mathrm{~K}$. The anisotropy remains up to $T_{\mathrm{N}}$, but the characteristic magnetization curves have significantly changed: along [100] the curve is almost linear up to saturation, whereas along [110] a jump at a lower field is still visible. Comparing the

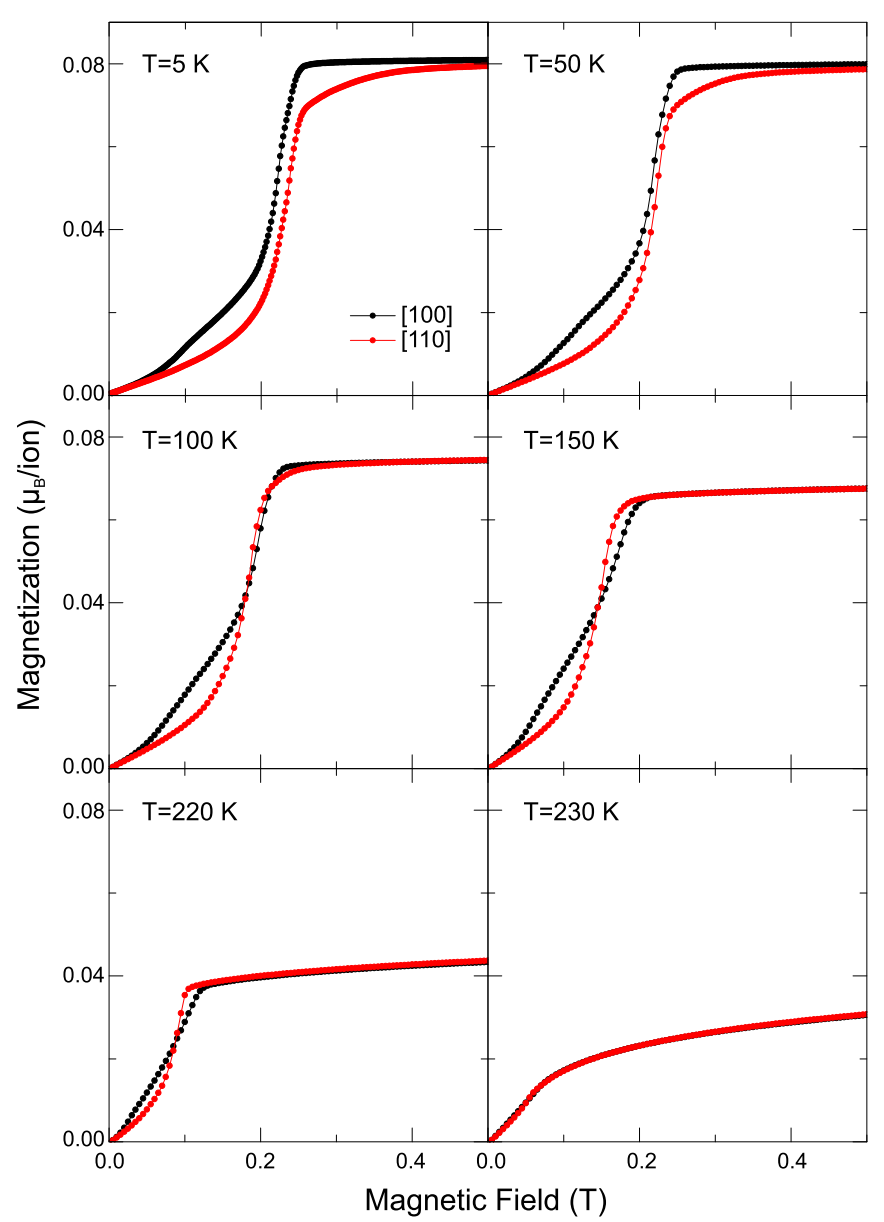

FIG. 8. Temperature evolution of the anisotropy in the magnetization as a function of magnetic field applied along [100] (black) and [110] (red), taken at $T=5,50,100,150,220$, and $230 \mathrm{~K}$.

data at $T=220 \mathrm{~K}$ with the calculated curves for anisotropic interlayer coupling $\Delta_{c}$ shown in Fig. 6(b), we conclude that the temperature-dependent in-plane anisotropy vanishes close to $T_{\mathrm{N}}$, while $\Delta_{c}$ remains. This is due to the coupling of the pseudospins to the lattice getting largely reduced as the magnetic order disappears [45]. Note that the contribution of $\Delta_{c}$ does not qualitatively modify the modeled curves for anisotropic magnetization at low temperature and has been included in Figs. 6(a) and 6(d).

Having established the necessity of both in-plane anisotropy $\Gamma_{1}$, which dominates magnetic anisotropy at low temperature, and anisotropic interlayer coupling $\Delta_{c}$, which becomes more important as temperature increases, we turn to their effect on the low-energy excitations in $\mathrm{Sr}_{2} \mathrm{IrO}_{4}$.

\section{MAGNETIC EXCITATIONS}

\section{A. Modeling}

We model the magnetic excitations using a code based on the SPINW library [46], using the Hamiltonian $H=$ $H_{\text {iso }}+H_{\text {ani }}^{(1)}+H_{\text {ani }}^{(2)}+H_{\text {sp-lat }}$ from Eqs. (1), (2), (4), and (5). The parameters $J_{1 \mathrm{c}}=16.4 \mu \mathrm{eV}, J_{2 \mathrm{c}}=-6.2 \mu \mathrm{eV}, \Delta_{c}=$ $0.02 J_{1 \mathrm{c}}$, and $\Gamma_{1}=2.7 \mu \mathrm{eV}$ are fixed from fits to the above magnetization data, and a quantum renormalization factor 


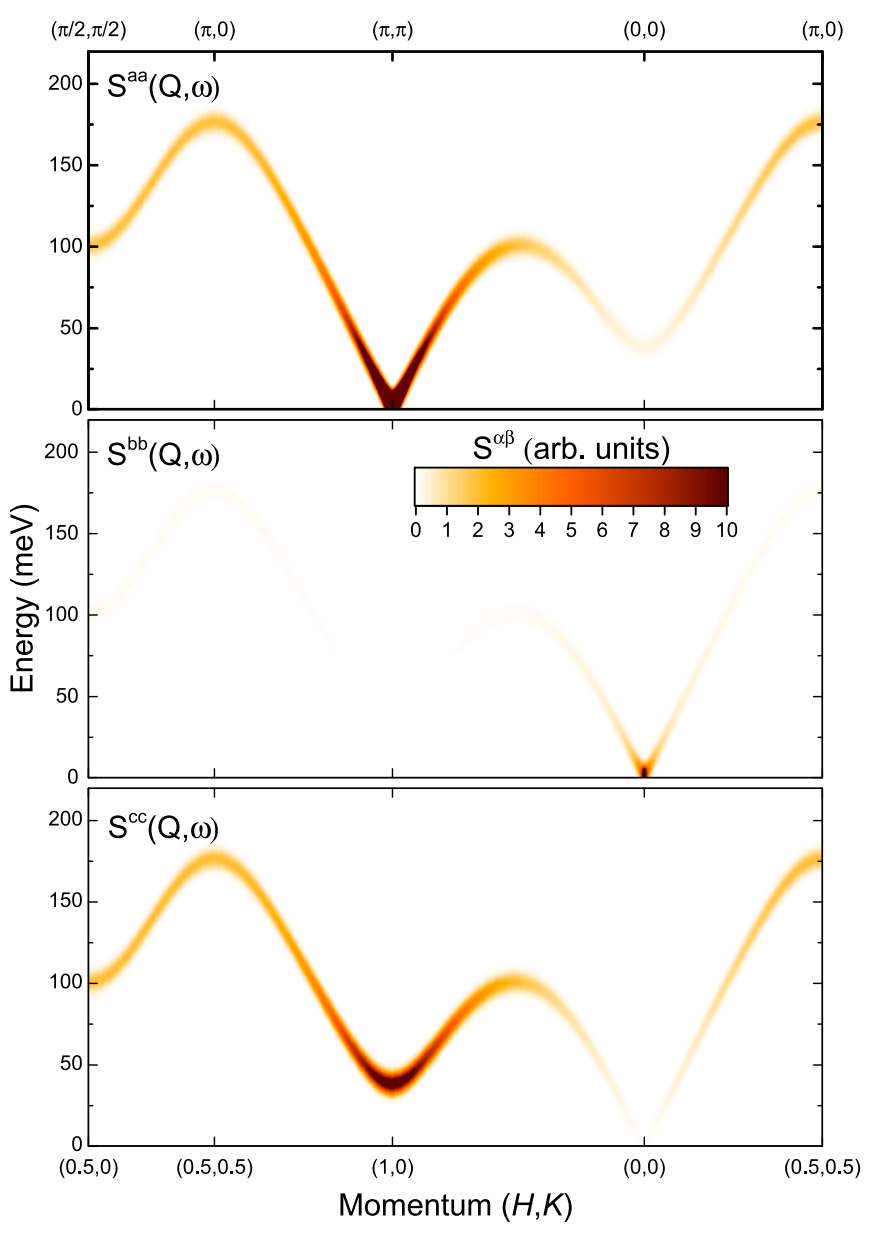

FIG. 9. Calculated dynamical spin structure factor as a function of momentum $Q$ and energy $\omega$ for spin components along the crystallographic directions $a$ (top), $b$ (middle), and $c$ (bottom). A Gaussian broadening $\delta E=10 \mathrm{meV}$ is used for clarity. The magnetic structure is chosen with the main component of the moments aligned along [100]. The in-plane momenta indicated on the top axis refer to the undistorted square-lattice unit cell, which is doubled for the magnetic unit cell indicated in the bottom axis in reciprocal-lattice units. The parameters used for the calculation are $J=57 \mathrm{meV}, J_{2}=$ $-16.5 \mathrm{meV}, J_{3}=12.4 \mathrm{meV}$, determined from RIXS measurements [15,16], and $\phi=13^{\circ}$, determined from neutron diffraction [26], which gives $D=28 \mathrm{meV}$. Taking $J_{z}=2.9 \mathrm{meV}$ results in an out-ofplane gap $\Delta_{\text {out }}=40 \mathrm{meV}$ consistent with our measurements shown in [36] (but larger than previously reported [16]).

$Z_{c}=1.67$ is applied based on recent calculations for the spin$1 / 2$ Heisenberg AFM on a square lattice [47]. The parameters for high-energy terms (known from previous RIXS spectra $[15,16])$ are listed in the caption of Fig. 9. The ground state has two degenerate solutions: antiferromagnetic moments along [100] with uudd stacking and along [010] with $\mathrm{lrrl}$ stacking. Given that there are four atoms per sublattice per unit cell, eight modes are expected: four in plane and four out of plane. Figure 9 shows the magnon dispersions and intensities for different spin components. As expected, the in-plane modes are almost degenerate and have a small gap at $(1,0)$ in the two-dimensional Brillouin zone, whereas the out-of-plane modes, also practically degenerate, have a larger

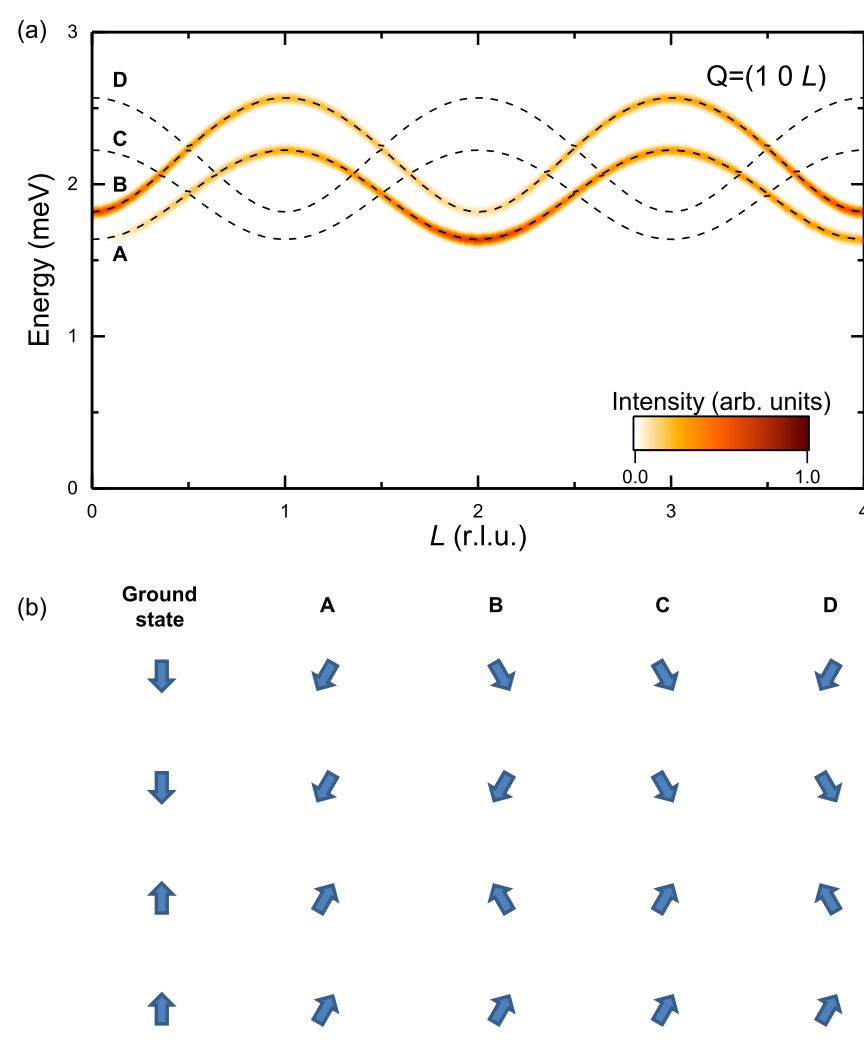

FIG. 10. (a) Calculated dynamical spin structure factor $S_{\text {perp }}$ perpendicular to $\vec{Q}$ close to the magnetic zone center as a function of $L$ in reciprocal-lattice units (r.l.u.) and energy, taking into account both magnetic twin domains present in the sample. A $\delta E=0.05 \mathrm{meV}$ Gaussian broadening is used for clarity. (b) Real-space representation for $Q=(100)$ of the four different magnon modes (A-D), where blue arrows represent the rotated net moments for each layer. The same parameters as in Fig. 9 were used.

gap, $\Delta_{\text {out }} \simeq 4 S \sqrt{2 J\left(D \tan \phi-J_{z}\right)}=40 \mathrm{meV}$. Note that the in-plane modes are visible in both $S_{a a}$ and $S_{b b}$ due to the canting of the moments.

Figure 10(a) shows the calculated low-energy excitations close to the magnetic zone center, where the orientation factor for inelastic neutron scattering has been taken into account for ease of comparison with the experiment. This calculation includes both magnetic twin domains present in the sample; however, the scattering from the domain with moments pointing along [100] is largely reduced due to the orientation factor. The splitting due to the effective interlayer coupling $j_{1 c}$ of the four in-plane modes can be clearly seen in the dispersions, with the bandwidth given by the effective coupling $j_{1 c}+2 j_{2 c}$ (as defined in the caption of Fig. 2). An increased splitting at $L=2 n$ for the upper two branches and at $L=2 n+1$ for the lower two is related to the anisotropy of the interlayer coupling $\Delta_{c}$. This gives a character for each mode related to the stacking patterns of the excited modes as shown in Fig. 10(b) for $Q=(100)$. Finally, the gap at $Q=(102)$ is due to the in-plane anisotropy $\Gamma_{1}$.

\section{B. Inelastic neutron scattering}

Inelastic neutron scattering (INS) is customarily used to study magnons in AFM materials, as the neutron cross 

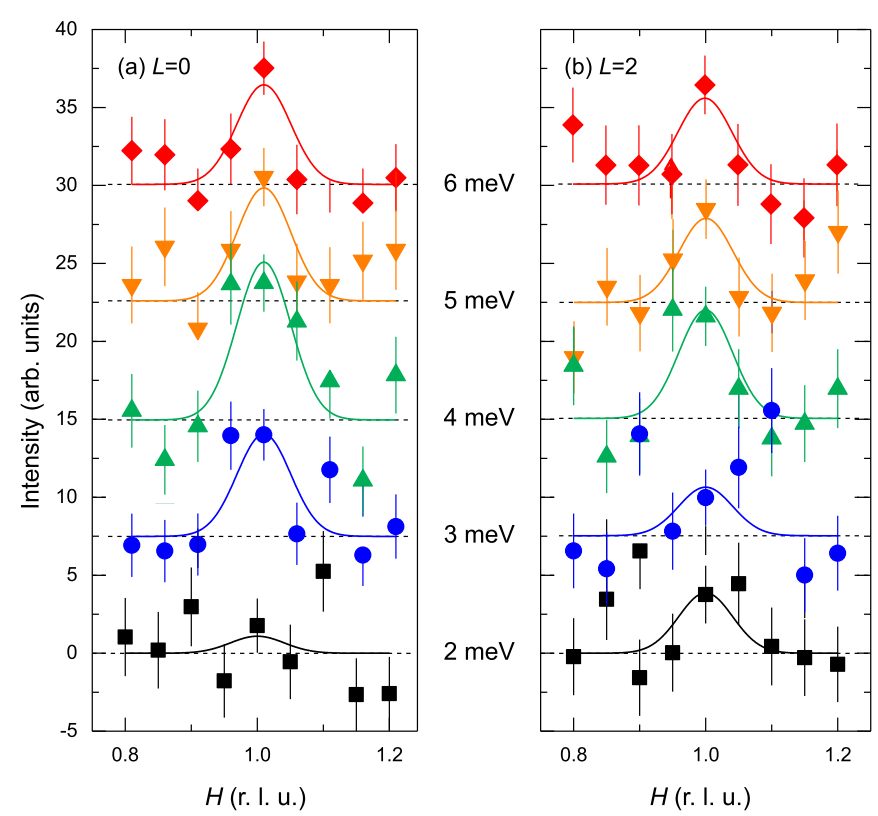

FIG. 11. Inelastic neutron scattering intensity as a function of $H$ in r.l.u. close to the magnetic zone center (a) (100) and (b) ( $\left.\begin{array}{lll}1 & 0 & 2\end{array}\right)$, measured for energy transfer $E$ from 2 to $6 \mathrm{meV}$. The intensity scale is approximately counts per $10 \mathrm{~min}$. Lines are results of constrained Gaussian fits with amplitudes and a common width as fitting parameters. A common background has been subtracted from the data, and a vertical offset is used for clarity.

section for magnetic scattering is sizable and sub-meV energy resolution is readily available. In the case of $\mathrm{Sr}_{2} \mathrm{IrO}_{4}$, neutron absorption from Ir nuclei is strong, which, coupled with a relatively small magnetic moment, makes INS measurements challenging. The experiment was conducted using the ThreeAxis instrument for Low Energy Spectrometry (ThALES) of Institut Laue-Langevin. A $\delta_{E}=0.15 \mathrm{meV}$ resolution and minimal extrinsic background were achieved using a $\mathrm{PG}(002)$ monochromator and analyzer and keeping $k_{f}=1.55 \AA^{-1}$ with cold $\mathrm{Be}$ as a filter. To maximize the magnon signal, an array of $\sim 300$ crystals coaligned on $\mathrm{Al}$ sheets was measured at magnetic zone centers (100) and (102), where the magnetic form factor and neutron absorption are manageable. In Fig. 11, $H$ scans across the magnetic zone center are shown. Below $2 \mathrm{meV}$ no magnetic signal can be discerned above the background level, whereas a magnon peak emerges at higher energies. This puts an upper bound on the magnon gap $\Delta_{\text {INS }}=2 \mathrm{meV}$. Note that at $2 \mathrm{meV}$ a slightly higher intensity is seen for $Q=(102)$ in comparison to $Q=(100)$, which might be related to seeing the A and B modes, respectively (Fig. 10).

\section{Resonant inelastic $x$-ray scattering}

In order to confirm the in-plane nature of the magnon mode in the INS spectra, we cross-checked the results using high-resolution RIXS measurements at the Ir $L_{3}$ edge ( $E=$ $11.215 \mathrm{keV}$ ) at the 27-ID of the Advanced Photon Source $[48,49]$. To achieve a $10 \mathrm{meV}$ energy resolution, an incident beam of $11.215 \mathrm{keV}$ was monochromated using a doublecrystal diamond high-heat load monochromator, and its band-

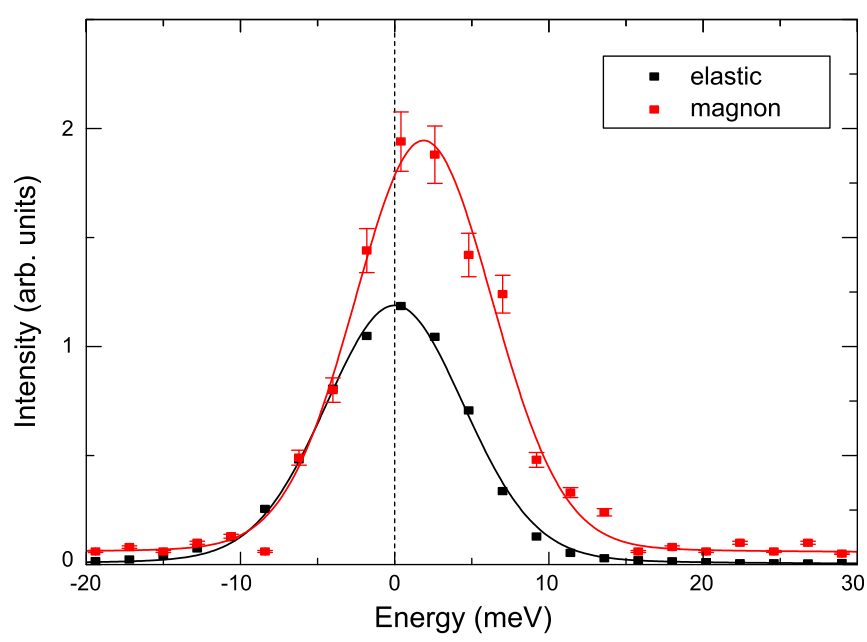

FIG. 12. High-resolution RIXS intensity as a function of energy for incoherent scattering of Scotch tape used as a reference (black) and the in-plane magnon mode in $\mathrm{Sr}_{2} \mathrm{IrO}_{4}$ (red) measured at $Q=$ (3 2 28.2) close to the magnetic zone center.

pass was further reduced to $8.9 \mathrm{meV}$ using a four-bounce symmetric Si (844) high-resolution monochromator. The beam was focused to a spot size of $10 \times 40 \mu \mathrm{m}^{2}$ FWHM $(V \times H)$ on the sample using a KB focusing mirror system. Scattered radiations from the sample are analyzed by a diced spherical quartz (309), which has an intrinsic bandpass of $3.7 \mathrm{meV}$ at the $\operatorname{Ir} L_{3}$ edge [48]. The in-plane magnon gap was probed at $Q=\left(\begin{array}{l}32 \\ 228.2\end{array}\right)$, where the sample surface is at a grazing angle to the incident beam and both $\left(\pi-\sigma^{\prime}\right)$ and $\left(\pi-\pi^{\prime}\right)$ probe only in-plane magnetic excitations. To have a resolution-limited magnon peak, a high momentum resolution is of particular importance given the relatively high spin-wave velocity; a 3-mm rectangular mask on the analyzer (on a 2-m-diameter Rowland circle) was used, giving a maximum $0.086^{\circ}$ divergence, which translates to $\delta q=0.0054$ r.l.u. in each in-plane direction and 0.4 r.l.u. in the out-of-plane direction at $Q=$ (3 2 28.2). Figure 12 shows the in-plane magnon measured at $Q=(3228.2)$, from which the in-plane anisotropy gap is estimated to be $2 \mathrm{meV}$ from the peak energy position. This is unequivocally smaller than the out-of-plane magnon gap seen in the standard geometry that was previously measured [16] (see [36] for a comparison of our measurements of the two magnon gaps).

The in-plane gap values measured with RIXS and INS are consistent with the calculated energies from the model, as well as the magnetic excitation emerging below $T_{\mathrm{N}}$ identified by Raman scattering in previous studies [29,30]; small differences are due to the interlayer couplings and dispersions along the $L$ direction.

\section{CONCLUSIONS}

To conclude, we have unambiguously shown that the equilibrium arrangements of pseudospins in the archetypal spin-orbit Mott insulator $\mathrm{Sr}_{2} \mathrm{IrO}_{4}$ cannot be explained by considering interactions among pseudospins alone and that their coupling to the lattice is essential for a quantitative description of the ground state. 
The lrrl and uudd stacking patterns of the net ferromagnetic moments peculiar to $\mathrm{Sr}_{2} \mathrm{IrO}_{4}$ provide a means to differentiate among different types of magnetic anisotropy as they undergo nontrivial changes under moderate applied fields: any fourfold symmetric magnetic anisotropy necessarily leads to the uurr stacking pattern stabilized in some range of field strength, the absence of which unequivocally implies reduced symmetry due to magnetostriction. We have directly confirmed that the magnetic structure evolves under applied field as expected in the pseudospin-lattice coupling model by using RMXS.

In the magnetization measurements, the critical fields of the metamagnetic transitions induced by fields applied along the [100] and [110] directions $\left(H_{c}^{100}<H_{c}^{110}\right)$ not only contain information on the symmetry of the magnetic anisotropy but also allow quantitative extraction of the magnitudes of interlayer couplings and anisotropy parameters. We have shown that the anisotropy of the nearest-neighbor interlayer coupling is responsible for the lifting of the degeneracy of $u d d u$ and $u u d d$ stacking patterns: for pseudospins along the $a(b)$ axis, lrrl (uudd) is stabilized. The interlayer coupling anisotropy is most manifest near the Néel temperature as the anisotropy due to the pseudospin-lattice coupling becomes suppressed with reduced moment size.

In the INS and RIXS spectra, the anisotropy due to pseudospin-lattice coupling is largely responsible for the inplane magnon gap. The measured gap is consistent with our model using the parameters extracted from the magnetometry.
The two anisotropic interactions uncovered in this study are of particular importance for determining the magnetic ground state of the system and give a complete description of the magnetism in $\mathrm{Sr}_{2} \mathrm{IrO}_{4}$.

The comprehensive understanding of the magnetic interactions and the magnetoelastic coupling in this archetypical model compound provides a firm basis for the interpretation of thermodynamic and spectroscopic data on other compounds with $4 d$ and $5 d$ valence electrons in various lattice geometries. For instance, the evidence for unconventional order parameters in iridates with various forms of disorder [32] should be critically reexamined in light of the crucial influence of pseudospin-lattice interactions on the magnetic ground state and excitations in a stoichiometric parent compound. Further, pseudospin-lattice coupling is expected to become particularly important for the phase behavior of Kitaev model materials [50], where pseudospin frustration leads to a large number of competing many-body states.

\section{ACKNOWLEDGMENTS}

We are grateful to $\mathrm{S}$. Park and $\mathrm{H}$. Kim for their assistance during the RMXS measurements. This work was supported by IBS-R014-A2. This research used resources of the Advanced Photon Source, a U.S. Department of Energy (DOE) Office of Science User Facility operated for the DOE Office of Science by Argonne National Laboratory under Contract No. DE-AC02-06CH11357.
[1] G. Khaliullin, Prog. Theor. Phys. Suppl. 160, 155 (2005).

[2] G. Jackeli and G. Khaliullin, Phys. Rev. Lett. 102, 017205 (2009).

[3] J. Chaloupka, G. Jackeli, and G. Khaliullin, Phys. Rev. Lett. 105, 027204 (2010).

[4] S. K. Choi, R. Coldea, A. N. Kolmogorov, T. Lancaster, I. I. Mazin, S. J. Blundell, P. G. Radaelli, Y. Singh, P. Gegenwart, K. R. Choi, S.-W. Cheong, P. J. Baker, C. Stock, and J. Taylor, Phys. Rev. Lett. 108, 127204 (2012).

[5] J. Chaloupka, G. Jackeli, and G. Khaliullin, Phys. Rev. Lett. 110, 097204 (2013).

[6] S. H. Chun, J.-W. Kim, J. Kim, H. Zheng, C. C. Stoumpos, C. D. Malliakas, J. F. Mitchell, K. Mehlawat, Y. Singh, Y. Choi, T. Gog, A. Al-Zein, M. M. Sala, M. Krisch, J. Chaloupka, G. Jackeli, G. Khaliullin, and B. J. Kim, Nat. Phys. 11, 462 (2015).

[7] B. J. Kim, H. Jin, S. J. Moon, J.-Y. Kim, B.-G. Park, C. S. Leem, J. Yu, T. W. Noh, C. Kim, S.-J. Oh, J.-H. Park, V. Durairaj, G. Cao, and E. Rotenberg, Phys. Rev. Lett. 101, 076402 (2008).

[8] B. J. Kim, H. Ohsumi, T. Komesu, S. Sakai, T. Morita, H. Takagi, and T. Arima, Science 323, 1329 (2009).

[9] J. Kim, D. Casa, M. H. Upton, T. Gog, Y.-J. Kim, J. F. Mitchell, M. van Veenendaal, M. Daghofer, J. van den Brink, G. Khaliullin, and B. J. Kim, Phys. Rev. Lett. 108, 177003 (2012).

[10] S. Fujiyama, H. Ohsumi, T. Komesu, J. Matsuno, B. J. Kim, M. Takata, T. Arima, and H. Takagi, Phys. Rev. Lett. 108, 247212 (2012).

[11] J. Bertinshaw, Y. K. Kim, G. Khaliullin, and B. J. Kim, Annu. Rev. Condens. Matter Phys. 10, 315 (2019).
[12] G. Khaliullin, Phys. Rev. Lett. 111, 197201 (2013).

[13] A. Jain, M. Krautloher, J. Porras, G. H. Ryu, D. P. Chen, D. L. Abernathy, J. T. Park, A. Ivanov, J. Chaloupka, G. Khaliullin, B. Keimer, and B. J. Kim, Nat. Phys. 13, 633 (2017).

[14] See recent works $[48,49,51]$ and references therein.

[15] H. Gretarsson, N. H. Sung, J. Porras, J. Bertinshaw, C. Dietl, J. A. N. Bruin, A. F. Bangura, Y. K. Kim, R. Dinnebier, J. Kim, A. Al-Zein, M. Moretti Sala, M. Krisch, M. Le Tacon, B. Keimer, and B. J. Kim, Phys. Rev. Lett. 117, 107001 (2016).

[16] D. Pincini, J. G. Vale, C. Donnerer, A. de la Torre, E. C. Hunter, R. Perry, M. Moretti Sala, F. Baumberger, and D. F. McMorrow, Phys. Rev. B 96, 075162 (2017).

[17] J. Kim, A. H. Said, D. Casa, M. H. Upton, T. Gog, M. Daghofer, G. Jackeli, J. van den Brink, G. Khaliullin, and B. J. Kim, Phys. Rev. Lett. 109, 157402 (2012).

[18] M. Moretti Sala, V. Schnells, S. Boseggia, L. Simonelli, A. Al-Zein, J. G. Vale, L. Paolasini, E. C. Hunter, R. S. Perry, D. Prabhakaran, A. T. Boothroyd, M. Krisch, G. Monaco, H. M. Rønnow, D. F. McMorrow, and F. Mila, Phys. Rev. B 92, 024405 (2015).

[19] X. Wan, A. M. Turner, A. Vishwanath, and S. Y. Savrasov, Phys. Rev. B 83, 205101 (2011).

[20] M. P. M. Dean, Y. Cao, X. Liu, S. Wall, D. Zhu, R. Mankowsky, V. Thampy, M. Chen, J. G. Vale, D. Casa, J. Kim, A. H. Said, P. Juhas, R. Alonso-Mori, J. M. Glownia, A. Robert, J. Robinson, M. Sikorski, S. Song, M. Kozina, H. Lemke, L. Patthey, S. Owada, T. Katayama, M. Yabashi, Y. Tanaka, T. Togashi, J. Liu, C. R. Serrao, B. J. Kim, L. Huber, C. L. Chang, 
D. F. McMorrow, M. Forst, and J. P. Hill, Nat. Mater. 15, 601 (2016).

[21] Y. K. Kim, O. Krupin, J. D. Denlinger, A. Bostwick, E. Rotenberg, Q. Zhao, J. F. Mitchell, J. W. Allen, and B. J. Kim, Science 345, 187 (2014).

[22] Y. K. Kim, N. H. Sung, J. D. Denlinger, and B. J. Kim, Nat. Phys. 12, 37 (2015).

[23] Y. J. Yan, M. Q. Ren, H. C. Xu, B. P. Xie, R. Tao, H. Y. Choi, N. Lee, Y. J. Choi, T. Zhang, and D. L. Feng, Phys. Rev. X 5, 041018 (2015).

[24] M. K. Crawford, M. A. Subramanian, R. L. Harlow, J. A. Fernandez-Baca, Z. R. Wang, and D. C. Johnston, Phys. Rev. B 49, 9198 (1994).

[25] C. Dhital, T. Hogan, Z. Yamani, C. de la Cruz, X. Chen, S. Khadka, Z. Ren, and S. D. Wilson, Phys. Rev. B 87, 144405 (2013).

[26] F. Ye, S. Chi, B. C. Chakoumakos, J. A. Fernandez-Baca, T. Qi, and G. Cao, Phys. Rev. B 87, 140406 (2013).

[27] J. G. Vale, S. Boseggia, H. C. Walker, R. Springell, Z. Feng, E. C. Hunter, R. S. Perry, D. Prabhakaran, A. T. Boothroyd, S. P. Collins, H. M. Rønnow, and D. F. McMorrow, Phys. Rev. B 92, 020406 (2015).

[28] S. Bahr, A. Alfonsov, G. Jackeli, G. Khaliullin, A. Matsumoto, T. Takayama, H. Takagi, B. Büchner, and V. Kataev, Phys. Rev. B 89, 180401 (2014).

[29] Y. Gim, A. Sethi, Q. Zhao, J. F. Mitchell, G. Cao, and S. L. Cooper, Phys. Rev. B 93, 024405 (2016).

[30] H. Gretarsson, J. Sauceda, N. H. Sung, M. Höppner, M. Minola, B. J. Kim, B. Keimer, and M. Le Tacon, Phys. Rev. B 96, 115138 (2017).

[31] D. H. Torchinsky, H. Chu, L. Zhao, N. B. Perkins, Y. Sizyuk, T. Qi, G. Cao, and D. Hsieh, Phys. Rev. Lett. 114, 096404 (2015).

[32] L. Zhao, D. H. Torchinsky, H. Chu, V. Ivanov, R. Lifshitz, R. Flint, T. Qi, G. Cao, and D. Hsieh, Nat. Phys. 12, 32 (2015).

[33] S. Di Matteo and M. R. Norman, Phys. Rev. B 94, 075148 (2016).

[34] A. J. Millis, P. B. Littlewood, and B. I. Shraiman, Phys. Rev. Lett. 74, 5144 (1995).
[35] O. Gunnarsson and O. Rösch, J. Phys.: Condens. Matter 20, 043201 (2008).

[36] See Supplemental Material at http://link.aps.org/supplemental/ 10.1103/PhysRevB.99.085125 for additional data and details on the experiments and data analysis.

[37] T. Takayama, A. Matsumoto, G. Jackeli, and H. Takagi, Phys. Rev. B 94, 224420 (2016).

[38] A. L. Chernyshev, Phys. Rev. B 72, 174414 (2005).

[39] M. D. Lumsden, B. C. Sales, D. Mandrus, S. E. Nagler, and J. R. Thompson, Phys. Rev. Lett. 86, 159 (2001).

[40] C. Wang, H. Seinige, G. Cao, J.-S. Zhou, J. B. Goodenough, and M. Tsoi, Phys. Rev. X 4, 041034 (2014).

[41] M. Nauman, Y. Hong, T. Hussain, M. S. Seo, S. Y. Park, N. Lee, Y. J. Choi, W. Kang, and Y. Jo, Phys. Rev. B 96, 155102 (2017).

[42] L. Fruchter, D. Colson, and V. Brouet, J. Phys.: Condens. Matter 28, 126003 (2016).

[43] T. Yildirim, A. B. Harris, A. Aharony, and O. Entin-Wohlman, Phys. Rev. B 52, 10239 (1995).

[44] V. M. Katukuri, V. Yushankhai, L. Siurakshina, J. van den Brink, L. Hozoi, and I. Rousochatzakis, Phys. Rev. X 4, 021051 (2014).

[45] H. Liu and G. Khaliullin, Phys. Rev. Lett. 122, 057203 (2019).

[46] S. Toth and B. Lake, J. Phys.: Condens. Matter 27, 166002 (2015).

[47] F.-J. Jiang and U.-J. Wiese, Phys. Rev. B 83, 155120 (2011).

[48] A. H. Said, T. Gog, M. Wieczorek, X. Huang, D. Casa, E. Kasman, R. Divan, and J. H. Kim, J. Synchrotron Radiat. 25, 373 (2018).

[49] J. Kim, D. Casa, A. Said, R. Krakora, B. J. Kim, E. Kasman, X. Huang, and T. Gog, Sci. Rep. 8, 1958 (2018).

[50] S. M. Winter, A. A. Tsirlin, M. Daghofer, J. van den Brink, Y. Singh, P. Gegenwart, and R. Valentí, J. Phys.: Condens. Matter 29, 493002 (2017).

[51] M. Moretti Sala, K. Martel, C. Henriquet, A. Al Zein, L. Simonelli, C. J. Sahle, H. Gonzalez, M.-C. Lagier, C. Ponchut, S. Huotari, R. Verbeni, M. Krisch, and G. Monaco, J. Synchrotron Radiat. 25, 580 (2018). 